\title{
$\angle S$ Research Square \\ The value of hydrogen and methane breath test in predicting colorectal polyps
}

\section{Xueyuan Zhang}

Guangdong Pharmaceutical University

\section{Xujuan Liu}

Guangdong Pharmaceutical University

\section{Wenrui Xie}

First Affiliated Hospital of Guangdong Pharmaceutical College

\section{Wenjing Ding}

North America Medical Education Fundation

Xingxiang He ( $\sim$ hexingxiang@gdpu.edu.cn )

First Affiliated Hospital of Guangdong Pharmaceutical College

\section{Research article}

Keywords: hydrogen and methane breath test, colorectal polyps, intestine barrier function, SIBO, LPS

Posted Date: July 28th, 2020

DOI: https://doi.org/10.21203/rs.3.rs-38671/v1

License: (c) (1) This work is licensed under a Creative Commons Attribution 4.0 International License. Read Full License 


\section{Abstract}

\section{Background}

To investigate the value of hydrogen and methane breath test in predicting colorectal polyps.

\section{Methods}

382 patients were enrolled in this study. They were divided into colorectal polyps group and Non polyps group as control. Hydrogen and methane breath test was used in both groups for small intestinal bacteria overgrowth (SIBO) diagnosis. All patients were examined by colonoscopy to investigate colorectal polyps. Diamine oxidase, D-lactate and Lipopolysaccharides (LPS) were measured in all patients to evaluate the intestinal barrier function.

\section{Results}

1) The age of colorectal polys group is significantly higher than control group $(P=0.000)$; the prevalence of colorectal polyps increases with age.

2) The prevalence of colorectal polys in male is significantly higher than female $(P=0.004)$;

3) Lactuloses breath test (LBT) was used for small intestinal bacteria overgrowth (SIBO) diagnoses. Prevalence of hydrogen dominate SIBO and methane dominate SIBO in colorectal polys group were significantly higher than control group $(P=0.000,0.013)$;

4) The prevalence of constipation was higher in colorectal polys group than control group $(P=0.023)$;

5) The peoples who had high hydrogen production peoples showed less intestinal barrier function damage and lower LPS level than peoples who had low hydrogen production $(P=0.029,0.049)$;

6) The sensitivity and specificity of SIBO is $75.5 \%$ and $51.0 \%$ respectively in SIBO diagnosis.

\section{Conclusion}

The occurrence of colorectal polyp increase with age, the year 46 is a cut off age for peoples need colonoscopy examination. Male has a higher prevalence than female. Intestinal hydrogen may play a role in prevent intestinal mucosa damage via its antioxidant function. SIBO shows a positive relationship with colorectal polyp. Hydrogen and methane breath test is a useful mass prescreening method for colorectal polyp risk.

\section{Background}

The colorectal polyps are the small clumps of proliferated cells on the colorectal mucosal. The person over 50 has a high risk to develop colorectal polyps. Most colorectal polyps are harmless, but over the 
time, some of them may develops into colorectal cancer. It is important to examine the colorectal polyps and remove them before they develop to cancerous. Screening tests can help find early stage colorectal cancer before it shows symptoms, to give a good chance of recovery.

The prevalence of colorectal polyps varies widely from different genders, ages, and races. Lieberman and colleagues reported the asymptomatic black men and women are more likely to have one or more polyps $>9 \mathrm{~mm}$ compared to whites ${ }^{[1]}$. The differences were especially striking among women. Wang and colleagues reported the prevalence of asymptomatic colorectal polyps is $\mathbf{2 7 . 4 \%}$ in Taiwan, the prevalence

of hyperplastic polyps and adenomatous polyps was $11.1 \%$ and $16.1 \%$, respectively ${ }^{[2]}$. They reported man had higher percentage adenomatous polyp than women $(71.9 \%$ vs. $28.1 \%, P=0.017)$.

Since the colorectal polyps are often no symptoms, it is hardly for asymptomatic peoples to looking for examination before they are in the later stages of the colorectal cancer which may fetal to life. Colonoscopy examination is an expensive and uncomfortable method to screen colorectal polyps and cancer. It is important to find a noninvasive and less expensive method to screen the peoples in the high risk persons.

The increased evidences showed the gut microbiome may play crucial role in carcinogenesis. Hydrogen and methane are the specific metabolite of intestinal bacteria fermentation. Hydrogen and methane breath test is a widely used as noninvasive technique to evaluate small intestinal bacteria overgrowth (SIBO) and methane-producing organisms in colorectal intestine.

We use lactulose hydrogen and methane breath test (LBT) to evaluate the small intestinal bacterial overgrowth and constipation in all patients. All subjects intestinal mucosal were examined by colonoscopy to evaluate the intestinal barrier condition. We also evaluate the prevalence polys with age and gender.

\section{Method}

\subsection{Study design}

We retrospectively reviewed patients in our hospital during the period from July 2017 to February 2019. 382 patients were recruited in this study ( 213 males and 169 females). The age range of subjects is from 22 to 92 , average age is $57 \pm 14$ years. All patients signed informed consent before the test. The study was approved by the ethics committee of the First Affiliated Hospital of Guangdong Pharmaceutical University.

All patients were tested by LBT and colonoscopy. The age, gender, LBT result, colonoscopy examination findings, intestinal barrier function and patients' history were recorded. Patients with colorectal polyps were in the colorectal polyps group and those with normal colorectal mucosa were in control group. All experiments were conducted in accordance with relevant guidelines and regulations. 


\subsection{Criteria for patient selection}

Inclusion criteria:

Colorectal polyp group: colorectal polyps (including inflammatory, proliferative, adenomatous, etc.) were observed under colonoscopy and confirmed by pathological biopsy.

Normal colorectal mucosa group: no colorectal polyp or other intestinal lesions were observed under colonoscopy.

Exclusion criteria:

(1) patients with acute intestinal infection;

(2) antibiotics used in recent 4 weeks;

(3) patients with severe heart, lung, brain and other diseases who are unable to tolerate colonoscopy examination;

(4) patients at risk of hypoglycemia;

(5) patients younger than 18 years old.

\subsection{Sibo and intestinal barrier function examination}

\subsubsection{SIBO examination}

The LBT was tested based upon North American Consensus' suggestion. Quintron BreathTracker SC model equipment was used to measure concentration of hydrogen, methane, and carbon dioxide. All patients were fast 12 hours and brushed teeth before tests. They took $10 \mathrm{~g}$ lactulose solution in warm water after taking fast breath test. Breath samples were collected in every 30 minutes until finished the test at the 150 minute after taking lactulose solution. No drink, food, exercise, and sleep was allowed during the tests.

SIBO criteria was based upon North American Consensus', suggestion. SIBO positive was diagnosed if breath hydrogen increased $\geq 20 \mathrm{ppm}$, or methane increased $\geq 10 \mathrm{ppm}$ above fasting baseline withing 90 minutes. SIBO was also diagnosed as positive if the combined value of hydrogen and methane increased $\geq 15 \mathrm{ppm}$ above the fasting baseline within 90 minutes based upon manufacture's suggestion.

\subsubsection{Intestinal barrier function examination}

Intestinal barrier function test: The test instruments and kits were purchased from Beijing Zhongsheng Jinyu Diagnostic Technology Co., Ltd. Blood samples were taken after 8 hours fast and storage in $4 \llbracket 6^{\circ} \mathrm{C}$ refrigerator for no more than 4 hours. Diamine oxidase, D-lactate and LPS were measured according to the instruction provided by the manufacturer. Patients' symptoms and signs were recorded 48 hours 
before the test. The normal reference value of diamine oxidase is $\leq 10 \mathrm{U} / \mathrm{L}, \mathrm{D}$-lactate $\leq 15 \mathrm{mg} / \mathrm{L}$, LPS is $\leq 20 \mathrm{U} / \mathrm{L}$. Any value above these numbers is considered as intestinal barrier function damage.

\subsection{Statistical methods}

All data were analyzed by using IBM spss22.0 statistical software. T test was used for comparison of two independent data. Four grid $C^{2}$ test was used to compare the qualitative data, $\mathrm{P}<0.05$ (bilateral) was considered as statistically significant.

\section{Result}

382 patients were enrolled in this retrospective study. All patients were conducted LBT for SIBO and examined by using colonoscopy for colonrectal polyps. Most of subjects had basic diseases (such as hypertension, type 2 diabetes, hyperlipidemia, fatty liver cirrhosis, functional constipation, chronic superficial gastritis, gastroesophageal reflux disease, peptic ulcer).

213 patients showed normal intestine mucosa. 169 patients had colorectal polyp (52 patients had inflammatory polyp, 29 had hyperplastic polyp, and 88 had adenomatous polyp). 136 polyps were $\leq$ $1.0 \mathrm{~cm}, 25$ polyps were between $1.0 \mathrm{~cm}$ and $2.0 \mathrm{~cm}, 8$ polyps were $\geq 2.0 \mathrm{~cm}$. 71 patients had single polyp, 98 patients had multiple polyp. 114 patients had polyp on the left side colon (including sigmoid colon, descending colon, and rectum) and 55 patients had polyp on other parts of the colon.

\section{Prevalence of polyp in age and gender}

The prevalence of colorectal polyp was significantly correlated with age. The average age of the colorectal polyp group was higher than control group $(P=0.000)$. The prevalence of the colorectal polyp was increased with age $(P=0.000)$. Male showed higher colorectal polyp prevalence than female $(P=0.004)$. The data present in the table 1 and table 2 .

Teble 1. The correlation of colorectal polys with age and gender

\begin{tabular}{|c|c|c|c|c|}
\hline & Polyp group & Control group & $t / c^{2}$ value & $P$ value \\
\hline & $N=169$ & $N=213$ & & \\
\hline Age $\ Y r \rrbracket$ & $62.14 \pm 11.663$ & $52.99 \pm 14.440$ & -6.855 & 0.000 \\
\hline $\operatorname{Sex}(M / F \rrbracket$ & 108(63.9\%)/61 (36.1\%) & $105(49.3) / 108(50.7)$ & 8.153 & 0.004 \\
\hline
\end{tabular}

Table 2. The prevalence of colorectal polys indifferent age groups 


\begin{tabular}{|c|c|c|c|c|}
\hline Age & Polyp \% & Normal mucosa & $c^{2}$ Value & P Value \\
\hline $18 \llbracket 45$ years & $11 \otimes 15.7 \% \bigotimes$ & $59 \rrbracket 84.3 \% \rrbracket$ & 34.527 & 0.000 \\
\hline $46 \rrbracket 60$ years & $65 \rrbracket 43.3 \% \square$ & $85 \rrbracket 56.7 \% \rrbracket$ & & \\
\hline \60years & $93 \rrbracket 57.4 \% \bigotimes$ & $69 \rrbracket 42.6 \% \rrbracket$ & & \\
\hline
\end{tabular}

\section{Correlation between colorectal polyp and SIBO}

SIBO was diagnosed based upon North American Consensus' suggestion and breath test manufacture's suggestion. 146 patients had SIBO in colorectal polyp group (146/169, 86.4\% 16 162 patients had SIBO in

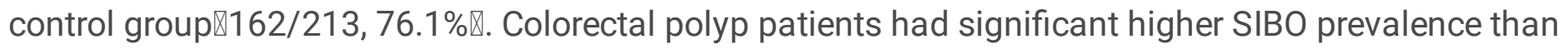
control group $(P=0.010)$.

Based upon small intestine hydrogen and methane production we divided the subjects into the following groups:

SIBO+: total LBT positive patients from both groups.

SIBO- : total LBT negative patients from both groups.

SIBO-M+: high methane production patients.

SIBO-M-: low methane production patients.

SIBO-H+: high hydrogen production patients.

SIBO-H-: low hydrogen production patients.

The prevalence of total SIBO patients, SIBO in high hydrogen production, and SIBO in high methane production in colorectal polyp group are significantly higher than that in control group. The data present on table 3.

Table 3 SIBO occurrence in colorectal polyp group and control group 


\begin{tabular}{|c|c|c|c|c|}
\hline & $\begin{array}{l}\text { Polyp group } \\
169\end{array}$ & $\begin{array}{l}\text { Control group } \\
213\end{array}$ & $C^{2}$ Value & P Value \\
\hline SIBO+ & 146囚86.4\%》 & 162ه76.1\%】 & \multirow[t]{2}{*}{6.558} & \multirow[t]{2}{*}{0.010} \\
\hline SIBO- & $23(13.6 \%)$ & $51(23.9 \%)$ & & \\
\hline SIBO-M+ & 120冈71.0\%》 & $125 \rrbracket 58.7 \% \rrbracket$ & \multirow[t]{2}{*}{6.219} & \multirow[t]{2}{*}{0.013} \\
\hline SIBO-M- & $49(29.0 \%)$ & $88(41.3 \%)$ & & \\
\hline SIBO-H+ & 101邓60.0\%】 & $86 \rrbracket 40.4 \% \rrbracket$ & \multirow[t]{2}{*}{14.174} & \multirow[t]{2}{*}{0.000} \\
\hline SIBO-H- & $68(40.0 \%)$ & 127 (59.6\%) & & \\
\hline
\end{tabular}

There is no significant correlation between SIBO and all types of polyps (including inflammatory polyp, hyperplastic polyp, adenomatous polyp). This finding suggests that the prevalence of SIBO is not correlation with the pathological classification of colorectal polyps.

\section{Correlation between colorectal polyp and constipation}

We evaluated the correlation between colorectal polyps and constipation. The constipation was diagnosed according to the "Rome IV" criteria. The patients who had diarrhea had been excluded from this study. The colorectal polyp group showed higher constipation prevalence than control group $(P=0.023)$. The data present in table 4.Table

4 incidence of constipation between polyp group and control group

\begin{tabular}{|c|c|c|c|c|}
\hline & Polyp group & Control group & $c^{2}$ value & $P$ value \\
\hline & $N=129$ & $N=160$ & & \\
\hline Constipation & $38 \rrbracket 29.5 \% \rrbracket$ & $29 \rrbracket 18.1 \% \bigotimes$ & 5.150 & 0.023 \\
\hline Normal bowel & $91 \rrbracket 70.5 \% \bigotimes$ & $131 \rrbracket 81.9 \% \bigotimes$ & & \\
\hline
\end{tabular}

\section{The correlation between breath hydrogen/methane and intestinal barrier function}

311 of the 382 patients were evaluated for intestinal barrier function by testing Diamine oxidase, Dlactate and LPS. We divided the 311 patients into four groups according to their small intestine hydrogen and methane production.

HMPG: The high methane production group includes all the patients whose breath methane was over 10 ppm above fasting baseline within 90 minutes.

LMPG: The low methane production group includes all the patients whose breath methane was less than 10 ppm above fasting baseline within 90 minutes. 
HHPG: The high hydrogen production group includes all the patients whose breath hydrogen was over 20 ppm above fasting baseline within 90 minutes.

LHPG: The low hydrogen production group includes all the patients whose breath methane was less than 20 ppm above fasting baseline within 90 minutes.

In this study, HHPG showed less intestinal barrier function damage than LHPG $\left(c^{2}=4.758 \bowtie P=0.029\right)$.

There is no significant difference between HMPG and LMPG in intestinal barrier function change $(P=0.188)$.

The correlation data between breath hydrogen/methane and intestinal barrier function present in Table 5 .

Table 5 Ccorrelation between intestinal barrier function and hydrogen/methane

\begin{tabular}{|c|c|c|c|c|}
\hline & \multicolumn{2}{|c|}{$\begin{array}{l}\text { intestinal barrier function damage } \\
\text { Yes No }\end{array}$} & $c^{2}$ value & $P$ value \\
\hline HMPG & $108 \rrbracket 52.9 \% \rrbracket$ & $96 \rrbracket 47.1 \% \rrbracket$ & 1.733 & 0.188 \\
\hline LMPG & $65 \rrbracket 60.7 \% \rrbracket$ & $42 \rrbracket 39.3 \% \square$ & & \\
\hline HHPG & $75 \llbracket 49.3 \% \rrbracket$ & $77 \rrbracket 50.7 \% \rrbracket$ & 4.758 & 0.029 \\
\hline LHPG & $98 \bowtie 61.6 \% \rrbracket$ & $61 \rrbracket 38.4 \% \rrbracket$ & & \\
\hline
\end{tabular}

The LPS level of HHPG is significantly lower than that of LHPG $(\mathrm{t}=1.974, P=0.049)$. The DAO and D-lac shows no difference between these two group patients. The data resent in table 6.

Table 6 Comparison of serological indexes of intestinal barrier between HHPG and LHPG

\begin{tabular}{|lllll|}
\hline & HHPG & LHPG & T value & P value \\
& $\mathrm{N}=152$ & $\mathrm{~N}=159$ & & \\
\hline DAO (U/L) & $5.89 \pm 7.26$ & $5.10 \pm 7.80$ & -0.924 & 0.356 \\
D-lac (mg/L) & $15.51 \pm 8.95$ & $15.95 \pm 8.90$ & 0.431 & 0.667 \\
LPS (U/L) & $8.42 \pm 8.79$ & $10.57 \pm 10.34$ & 1.974 & 0.049 \\
\hline
\end{tabular}

\section{The value of SIBO in predict colorectal polyp}

We divided the SIBO positive patients into three groups based upon their hydrogen and methane production, then evaluated the sensitivity and specificity of each group. The data present in the table 7 .

Table 7 The sensitivity and specificity of hydrogen and methane in predict colorectal polyp 


\begin{tabular}{|lllllll|}
\hline & Subject No. & Area Under Curve & P Value & $95 \% \mathrm{Cl}$ & Sensitivity & specificity \\
\hline SIBO-H+ & 59 & 0.454 & 0.373 & $0.353-0.555$ & $39.8 \%$ & $51.0 \%$ \\
\hline SIBO-M+ & 117 & 0.525 & 0.568 & $0.440-0.610$ & $63.2 \%$ & $41.7 \%$ \\
\hline SIBO-MH+ & 128 & 0.632 & 0.001 & $0.556-0.708$ & $75.5 \%$ & $51.0 \%$ \\
\hline
\end{tabular}

SIBO-H+囚Hydrogen dominate SIBO.

SIBO-M+: Methane dominate SIBO.

SIBO-MH+: Combination of hydrogen and methane SIBO.

\section{Discussion}

Colorectal cancer quickly increases China. It is the third common cancer and fifth mortality cancer in China. Chronic colorectal inflammation and adenomatous polyp have high risks in colorectal cancer. The colorectal cancer may be prevented by screening high risk persons by using colonoscopy examination. The colorectal polyps are often ignored because the symptoms are often not obvious. It is often misdiagnosed as anal diseases such as hemorrhoids. The colonoscopy examination is not suitable for mass screening since uncomfortable, time consuming, and expensive. It is important to find a comfortable and less expensive way for mass prescreening before colonoscopy examination.

Our study confirms colorectal polyp increase with age. Peoples over aged 46 have significant higher prevalence of colorectal polyp than younger peoples ( $43.3 \%$ vs. $15.7 \%, P=0.000)$. Over half of the population aged over 60 had colorectal polyp in our study. Male had higher prevalence of colorectal polyp than female $(63.9 \%$ vs. $36.1 \%, P=0.004)$. The finding is consistent with the previous study by Wang and colleagues $^{[2]}$. We suggest the year 46 is a cut off age for colonoscopy examination. A suitable mass prescreen method is required before colonoscopy examination.

Adenomatous polyp is the precursor lesions for colorectal cancer. We found 88 patients had adenomatous polyp in total 382 patients $(23.3 \%)$. This finding agreed with Giacosa and colleagues' study $^{[3]}$. They reported adenoma prevalence is more than $25 \%$ in colonoscopy studies.

\section{Correlation between colorectal polyp and SIBO}

In recent years, increasing studies have been revealed the occurrence and development of colorectal polyp have close relationship with the change of intestinal flora. Chen and colleagues ${ }^{[4]}$ compared the intestinal mucosa bacterial composition between normal colorectal individuals and colorectal adenoma individuals by molecular fingerprint and clone sequencing technology. Their result showed a significant difference between these two populations. They suggested adherent bacteria might play an important role in adenoma and colorectal cancer development. The changes of the intestinal flora composition and structure of the adherent bacterial community might contribute to the occurrence and development of 
adenomatous polyp. Mangifesta and colleagues ${ }^{[5]}$ used 16S rRNA gene sequencing revealed the composition of intestinal polyp microbial community. In a meta-analysis, it reported in addition to genetics, diet, lifestyle, obesity, drinking and smoking, intestinal microflora played a very important role in the occurrence of colorectal cancer and colorectal polyp. Intestinal flora and its metabolites might be involved in the initiation or development of colorectal polyp or tumors through various mechanisms, including the production of free oxygen radicals and other gene toxins, phenolic compounds, indoles, etc.

We found SIBO had a correlation with polys $(P=0.010)$, either hydrogen dominate SIBO or methane dominate SIBO had significant correlation with colorectal poplys $(P=0.000,0.013)$. Our finding suggested intestinal Methanogenic bacteria might play a role in colorectal polyp development. We didn't evaluate the correlation between SIBO and colorectal polyp pathological types, diameter, size, number and location. This is the first study to investigate the correlation between SIBO and colorectal polyps.

We found that the incidence of constipation in the colorectal polyp group was significantly higher than control group ( $29.5 \%$ vs $18.1 \%, P=0.023)$, and the colorectal polyp group showed higher methane production than control group $(71.0 \% \mathrm{vs}, 58.7 \%, \mathrm{P}=0.013)$. Waqar and Rehan ${ }^{[6]}$ reported methane prolonged oro-cecal transit time (OCTT) and raised the intestine contraction amplitude, slowing peristalsis, resulting in constipation. Liu $^{[7]}$ reported that constipation can significantly increase the incidence of colorectal polyps, but did not significantly increase the incidence of colorectal cancer even it is a risk factors for colorectal cancer. Comstock and colleagues ${ }^{[8]}$ reported inflammatory cytokines level in patients with colorectal polyp was higher than that in patients without colorectal polyp. Their finding suggested colorectal polyp are associated with chronic intestinal inflammation. The increase of cytokines and the decrease of antioxidants will lead to oxidative stress, delay gastrointestinal motility and lead to SIBO.

\section{Hydrogen may protect intestinal mucosal from inflammation}

In this study, we found LPS level of HHPG is significantly lower than LHPG ( $t=1.974, P=0.049)$. LPS is known as lipoglycans and endotoxins, found in the outer membrane of gram-negative bacteria in intestine. Less LPS level in blood plasma can be considered less intestinal mucosa damage in HHPG than LHPG. Bilinski and colleagues ${ }^{[9]}$ reported neoplastic colon polyp displaying increased acute and chronic inflammation comparing to normal and adjacent non-dysplastic colonic mucosa. The adenoma polyp' size correlated with degree of acute and chronic inflammation. It is well known that oxidative stress plays an important role in intestine inflammation and can lead to damages of the intestinal mucosa and bacterial metabolite invasion, such as LPS. Hydrogen has the functions of selective antioxidant, anti-inflammatory, inducing gene expression and gas signal modulation. Chen and colleagues reported $^{[4]}$ molecular hydrogen was a potent antioxidant and able to protect organs from oxidative stress injuries. Lactulose administration significantly induced hydrogen production might help to prevent the development of dextran sodium sulfate-induced colitis and alleviate oxidative stress in the colon. Our finding shows agreement with their reports. The persons who produced higher hydrogen had 
less intestinal mucosa damage. Hydrogen breath test may have potential role in evaluate the intestinal mucosa inflammation.

\section{The value of hydrogen and methane breath in prescreening colorectal polys}

It is important to find a convenience method for mass prescreening the people who has risk of colorectal polyp and cancer development. We evaluate the colorectal polyp prediction value of LBT SIBO positive patients based upon their hydrogen and methane production. The result showed hydrogen dominate SIBO had a low sensitivity and specificity. It may not a good method in predict the occurrence of colorectal polyp. The Methane dominate SIBO has a moderate sensitivity and specificity, may also not a proper method to the occurrence of colorectal polyp. The SIBO patients who had both high hydrogen and methane had $75.5 \%$ sensitivity and $51.0 \%$ specificity in predict colorectal polyp. It suggests high hydrogen and methane LBT may have a predict value in colorectal polyps mass prescreening before colonoscopy examination.

Summarize the findings in this study, colorectal polyp has a correction with constipation and high methane production. The high methane excretion on breath test is association with clinical constipation. The North America Consensus suggested breath test is useful in the diagnosis of methane associated constipation. Our study confirmed the relationship of high methane production, constipation, and colorectal polyp. Based upon our knowledge this is the first study to evaluate the predict value of hydrogen and methane breath test in colorectal polyp occurrence.

Hydrogen and methane breath test is a noninvasive and less expensive test. It shows a clinical value in predict colorectal polyp for mass prescreening. The findings from this study suggest hydrogen and methane breath test may help to reveal the colorectal polyp in the early stage and help in colorectal cancer prevention. It may also have a value in helping reduce medical care expenses and save medical resources.

\section{Conclusion:}

The prevalence of colorectal polyp increases with age. Male has a higher prevalence than female in the same age group. Intestinal hydrogen may play a role in prevent intestinal barrier function damage via its antioxidant function. Both hydrogen and methane dominated SIBO have a correlation with colorectal polyp. Hydrogen and methane breath test has value for mass prescreening in discovering the colorectal polyp in the early stage and cancer prevention.

This is a retrospective study. The random controlled double blind study needs to be done in a large scale to re-evaluate the findings from this study. Further studies need to be done to investigate the impact of hydrogen on intestine inflammation, intestinal permeability, LPS, and colorectal polyp.

\section{Abbreviations}




\begin{tabular}{|ll|}
\hline Abbreviation & Full English \\
\hline SIBO & small intestinal bacteria overgrowth \\
\hline LBT & Lactuloses breath test \\
\hline LPS & Lipopolysaccharides \\
\hline DAO & diamine oxidase \\
\hline OCTT & D-lactate \\
\hline M & oro-cecal transit time \\
\hline H & methane \\
\hline MH & $\begin{array}{l}\text { methane and hydrogen } \\
\text { HMPG }\end{array}$ \\
\hline LMPG & $\begin{array}{l}\text { The high methane production group includes all the patients whose breath methane } \\
\text { was less than } 10 \text { ppm above fasting baseline within } 90 \text { minutes }\end{array}$ \\
\hline HHPG & $\begin{array}{l}\text { The high hydrogen production group includes all the patients whose breath hydrogen } \\
\text { was over } 20 \text { ppm above fasting baseline within } 90 \text { minutes }\end{array}$ \\
\hline LHPG & $\begin{array}{l}\text { The low hydrogen production group includes all the patients whose breath methane } \\
\text { was less than } 20 \text { ppm above fasting baseline within } 90 \text { minutes }\end{array}$ \\
\hline
\end{tabular}

\section{Declarations}

1. Ethics approval and consent to participate[The study protocol was approved by the institutional review board and Ethics Committee of The First Affiliated Hospital of Guangdong Pharmaceutical University.

2. Consent to publishnThe work described has not been submitted elsewhere for publication in whole or in part, and all the authors listed have approved the manuscript that is enclosed.

3. Availability of data and materials: All data and material are available upon request.

4. Competing interestsロThere are no any ethical/legal conflicts involved in the article.

5. Funding: This research received no specific grant from any funding agency in the public, commercial, or not-for-profit sectors.

6. Authors' contributions $\square$ In this manuscript, $X Y$ Zhang collected data and completed the first draft, XJ Liu completed statistic and analysis for data, WR Xie designed the report. WJ Ding and XX He revised the manuscript, and XX He supervised all the work. All authors have read and approved the manuscript.

7. Acknowledgements: Not applicable. 


\section{References}

1. Lieberman D-A, Holub J-L, Moravec M-D, et al. Prevalence of colon polyps detected by colonoscopy screening in asymptomatic black and white patients[J]. JAMA. 2008;300(12):1417-22.

2. Wang F-W, Hsu P-I, Chuang H-Y, et al. Prevalence and risk factors of asymptomatic colorectal polyps in taiwan[J]. Gastroenterol Res Pract, 2014, 2014985205.

3. Giacosa A, Frascio F, Munizzi F. Epidemiology of colorectal polyps[J]. Tech Coloproctol. 2004;8(Suppl 2s):243-s247.

4. Chen X, Zhai X, Shi J, et al. Lactulose mediates suppression of dextran sodium sulfate-induced colon inflammation by increasing hydrogen production[J]. Dig Dis Sci. 2013;58(6):1560-8.

5. Mangifesta M, Mancabelli L, Milani C, et al. Mucosal microbiota of intestinal polyps reveals putative biomarkers of colorectal cancer[J]. Sci Rep. 2018;8(1):13974.

6. Bin Waqar-SH. Rehan A. Methane and Constipation-predominant Irritable Bowel Syndrome: Entwining Pillars of Emerging Neurogastroenterology[J]. Cureus. 2019;11(5):e4764.

7. Liu B. Correlation between chronic constipation and colorectal neoplasms[J]. Zhonghua Wei Chang Wai Ke Za Zhi. 2017;20(3):255-7.

8. Comstock S-S, Xu D, Hortos K, et al. Association of serum cytokines with colorectal polyp number and type in adult males[J]. Eur J Cancer Prev. 2016;25(3):173-81.

9. Bilinski C, Burleson J, Forouhar F. Inflammation associated with neoplastic colonic polyps[J]. Ann Clin Lab Sci. 2012;42(3):266-70. 